\title{
Factorial effects in the categorization of externally distributed stimulus samples'
}

WAYNE LEE AND THOMAS R. ZENTALL

UNIVERSITY OF CALIFORNIA, BERKEI.EY

On each trial a sample point randomly drawn from one of two normal probability distributions was exhibited to $S$, who had to guess whether the sample was a "1" or a "2." He was then given feedback, which was determined by which of the two distributions the sample point derived from. Two continua were employed: dot position on a file card, and grayness of square in. patches. Three levels of $d$ ' were employed. The function giving the probability of a response "1" for different sample values was sharper for the higher $d$ 'conditions, and for the dot position continuum. Cessation of feedback resulted in improved performance for the low d' condition. Incentive payoff, confidence ratings, and experimenter had virtually no effects on task performance.

A number of previous studies have considered the manner in which $S$ categorizes a sample point that came from one of two possible normal probability distributions (Lee, 1963; Lee \& Janke, 1964; 1965). Such a distribution was called an externally distributed stimulus, since it is analogous to a stimulus such as a tone or attitude statement which is presumed in detection theory and Thurstone theory to give rise internally on each presentation to a sample point from a probability distribution. In the present case, however, such a sampling is done from a distribution which has variance external to $S$. This allows more information to be obtained on how $\mathrm{S}$ handles such a problem.

The present experiment was designed to determine the effects of a number of variables on the manner in which Ss perform such categorizations. The following variables were employed: (a) presence or absence of monetary incentive for efficient performance, (b) experimenter, (c) inclusion of a confidence rating in the response or not, (d) discriminability between distributions (d'), (e) type of stimulus continuum, and (f) presence or absence of feedback. The experimenter and confidence rating factors were included as technical checks, and not in the expectation that they were important determiners of task performance. Previous research has indicated that stimulus continuum was such a determiner, but this result was established only for one level of $d^{\prime}$. The present experiment allowed investigation of task performance across widely differing levels of $\mathrm{d}$ ', and a test of the generality of a continuum effect at different d' levels. A payoff effect was anticipated, on the basis of previous effects reported in binary prediction and in psychophysics. One could reason that the cessation of feedback could result in an increment or decrement in performance merit. Experimentation is necessary to resolve this issue.

\section{Method}

Stimuli. Stimuli were prepared in a manner similar to the preparation described in Lee \& Janke (1964). Three levels of d' were used: $1.25,2.25$, and 3.5 (d' is the distance between the two normal distributions in units of their common standard deviation). Two continua were employed at each $d^{\prime}$ ' level: dotposition along a $5 \times 8$ in. file card, and grayness of a square in. patch of paper pastec on a $3 \times 5$ in. file card. For each of these six conditions, 400 stimulus cards were prepared.

Each set of 400 stimulus cards was composed of two decks of 200 cards each. Each deck contained 100 sample points from each of the two probability distributions. The sample point positions were chosen so that each sample point represented $1 \%$ of the area of one of the two normal distributions. The 100 cards for each of the two distributions were then randomized together to form a deck of 200 cards. The randomization was different for each of the 200 card decks forming a set of 400 cards, and differed across d' levels, but was the same across the two continua for the same (first or second) deck and same d'.

Dots could occur at any of 42 equally spaced positions along an imaginary horizontal line on the $5 \times 8$ in. file card, but occurred no closer than 1 in. from each vertical edge. Each gray sample was from one of 14 possible gray papers. The actual distances between distribution means for the dot position dimension for the three $d^{\prime}$ conditions, from low to high, were 1.17, 1.82, and 2.42 in., respectively. Correspondence between the two continua was obtained by letting the lightest gray refer to the first three of the 42 possible dot positions, the next lightest gray to the next three dot positions, etc. The means of the two distributions for the dot position continuum were equally distant from the vertical centerline of the card.

Procedure. The Ss were 96 students, men and women, at the University of California in Berkeley who were sent by the University PlacementOffice. All Ss received a basic hourly rate, and, in addition, half the Ss were told that they would receive $\$ .01$ for every stimulus card which they correctly categorized above the chance level of 200 correct responses in 400 trials.

Ss were mun two at a time. Each pair of Ss was placed in one of the 48 conditions of the experiment determined by factorial combination of d' (three levels), incentive payoff or not (two levels), experimenter (two levels, male graduate students in psychology), type of continuum (two levels), and confidence ratings required or not (two levels). They were told that they were 
going to see a series of cards with dots (or gray papers) on them and that each card was either a "1" or a "2." Their job was to state which they thought each card was. They were told not to worry about getting each one correct and that they would improve with experience. On each trial $\mathbf{E}$ showed a card to the Ss. After seeing a card, the Ss wrote down their guesses as to the correct answer, "1" or " $2, "$ on an answer sheet. The $E$ told them whether the card actually was a "1" or a "2," depending on which stimulus distribution the card came from. The samples from the stimulus distribution to the left for the dot position continuum were called " 1, " and those from the right-hand distribution were called "2." For the gray continuum the distribution with the mean in the lighter grays was called "1," the distribution with the mean in the darker grays was called "2." After seeing the first 300 stimulus cards, Ss were told that they would no longer receive feedback but that they should continue responding according to their best guess as before.

After finishing 400 trials, Ss were asked to estimate the centers, or average positions, of those samples which were called " 1 "' by $E$, and of those which were called "2." Those Ss who had seen the dot continuum made their estimates with pencil on $5 \times 8$ in. file cards similar to the ones the sample points had been on. Those Ss who had seen the gray continuum were shown a set of cards with the different gray shades that had been used lined up in order, together with a nearwhite and a near-black, making 16 samples altogether. Ss picked out the average grays from this series.

\section{Results}

Choice likelihood function. Figure 1 shows the mean probability that Ss responded " 1 " as a function of sample position along the continuum for each d' condition for the last 200 trials. This will be called the choice likelihood function. In Fig. 1 the gray and dot continua are averaged together, using the correspondence of three dot positions to each gray shade, as previously explained. According to decision theory, the choice likelihood function should be a step function with a probability of 1.0 up to a cutoff point, and of 0.0 to the right of the cutoff point. Previous work has shown that such is generally not the case, even considering limited powers of sensory discrimination. An alternative response mode which seems more reasonable from the viewpoint of statistical learning theory is for Ss to respond probabilistically at each stimulus position, and with the same probability as the reinforcement probability at each position. This is called micromatching (Lee, 1963). If Ss performed in this manner the choice likelihood function would coincide with the reinforcement likelihood function, which gives the probability that $\mathrm{E}$ will give "1" as the correct response as a function of sample position.

An analysis of variance was performed on all Ss to determine whether, as it appeared, the choice likeli-
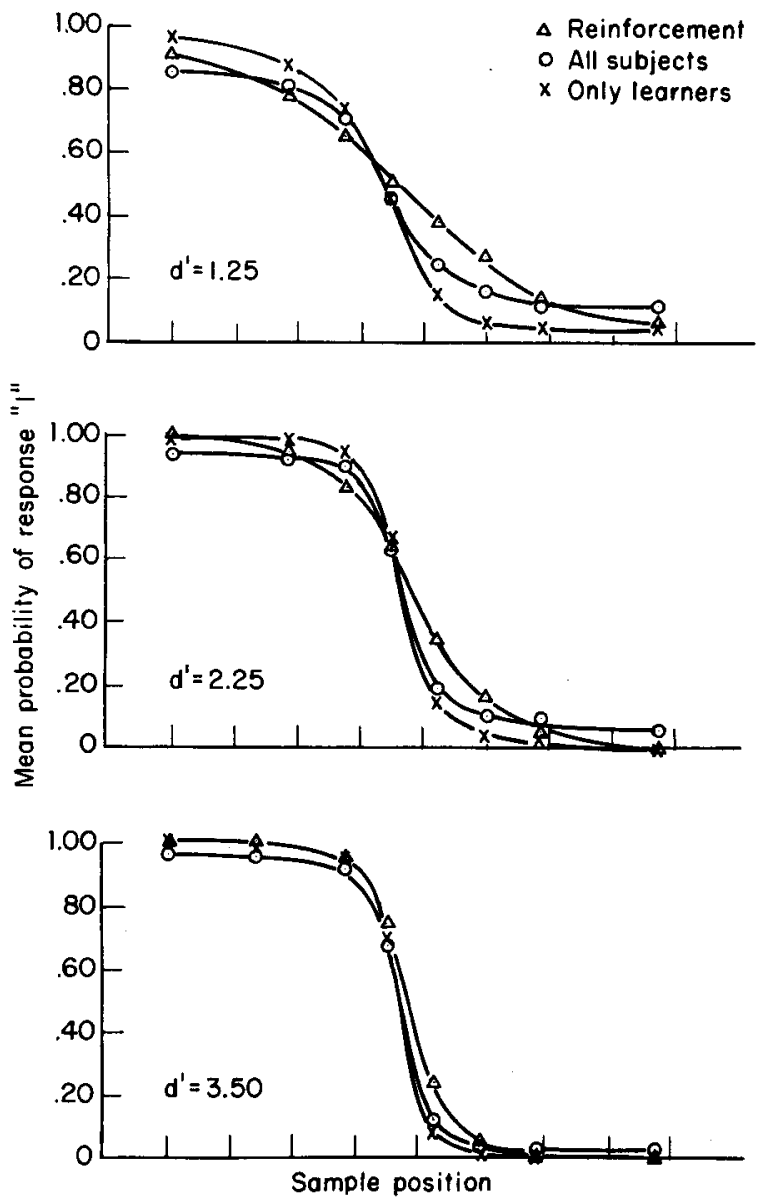

Fig. 1. Mean probability of response " 1 " as a function of sample position, and mean reinforcement probability for the same positions.

hood function was sharper the higher the d', in accordance with sucl. . distinction for the reinforcement likelihood functions. For this analysis all factors of the experiment were considered fixed, and the feedback factor was the only repeated measures factor. The criterion was the distance between the .75 and the .25 points on the choice likelihood function. It was not possible to determine such a figure for some Ss, since their functions were too flat or irregular (non-monotonic). Those Ss for whom such a figure could be determined were called learners, and the other Ss were called non-learners. There were a total of 14 nonlearners out of $96 \mathrm{Ss}, 9,3$, and 2 in tive low, middle, and high $d^{\prime}$ conditions respectively. In spite of the fact that the $.75-.25$ distance could not be obtained for all $\mathrm{Ss}$, it was desirable to have some figure of merit in order to perform an analysis of variance on all Ss. To this end an arbitrary distance was taken for those Ss who did not learn, which was considerably larger than the distance for any $S$ who did learn. Because these large distances resulted in skewed distribution of scores 
a transformation was used to help symmetrize the data. The transformation was the square root of: 100 over the distance. The following effects were statistically significant: $d^{\prime}(F=17.9, \mathrm{df}=2 / 48, \mathrm{p}<.001)$; continuum $(F=4.3, \mathrm{df}=1 / 48, \mathrm{p}<.05)$; trials $(F=7.5, \mathrm{df}=1 / 48$, $\mathrm{p}<.01) ; \mathrm{d}^{\prime} \mathrm{x}$ trials $(\mathrm{F}=3.4, \mathrm{df}=2 / 48, \mathrm{p}<.05)$. In addition, a fourth-order interaction was significant at the .05 level; however, considering the lack of a theoretical rationale for this, together with the fact that a very large number of $F$ ratios for higher interactions were calculated, this effect was not taken seriously. The d' effect was expected, and verifies the impression from the graphs that the higher $d^{\prime}$ choice functions were sharper than the lower d' function; the middle d' condition was about midway between the extremes. The dot position continuum had a sharper choice function than the gray continuum, which confirmed the previous finding of Lee \& Janke (1964). The fourth 100 trials, without feedback, had sharper choice likelihood functions than the third 100 trials, but only for the $d^{\prime}=1.25$ condition.

The lack of effect of payoff motivation was the only surprising result of this analysis. Since it appeared that the non-learners were equally divided among the two payoff groups, and it was thought there might be a depressing effect on the payoff group by those Ss who did not solve the task, a supplementary analysis of variance was done on learners only. The design was collapsed across the factors of experimenter and confidence rating in order to bolster the number of Ss in a cell, and the analysis was performed on payoff, feedback, $d '$, and continuum. One cell had four individuals who did not solve the problem, leaving only four Ss. All other cells had at least five Ss who did learn to perform the task. Therefore five learners were randomly selected for each cell, except that for the cell with only four learners a pseudo-fifth $S$ was entered with the means for the four learners for the third and fourth 100 trials. The df were reduced accordingly. The analysis of variance on the (untransformed, now) distance between the .75 and the .25 points of the choice function yielded the following significant effects: $d$ ' $(F=16.1, \mathrm{df}=2 / 47, \mathrm{p}<.001) ;$ trials $(\mathrm{F}=6.2, \mathrm{df}=1 / 47$, $\mathrm{p}<.05) ; d^{\prime} x$ trials $(F=6.0, d f=2 / 47, p<.01)$. Thus there was no payoff effect even considering just learners. The significant effects agree with those of the previous analysis, except that the effect for continuum did not reach significance.

It is worth considering whether the increase in the sharpness of the choice likelihood function from the third to the fourth 100 trials for the $d^{\prime}=1.25$ condition might have been due to learning. That is, at the beginning, Ss have essentially a flat choice likelihood function, since they have no idea where a "1" or a "2" is apt to be located. With trials, the function becomes sharper, and approaches an symptotic level. If Ss were still learning for the lowest $d^{\prime}$ condition, during the third 100 trials, and thereafter remaine unchainged when feedback was eliminated, they would have a sharper function for the fourth 100 trials than for the third 100. The evidence suggests that such was not the case. In the first place, previous studies in this series indicate that for successive trials of a single task, Ss reach an apparently asymptotic level of performance by Trial 200 or earlier. In addition, the sharpness for each $S$ of the choice function for the lowest d' condition was compared for the Trials 101-200 versus 201-300, and again for Trials 201-300 versus 301-400. The sign test indicated an increase in sharpness for the latter comparison, verifying the analysis of variance, but no change for the former comparison, indicating no learning in the 200 trials previous to cessation of feedback.

Center estimates. An analysis of variance was performed on the distance between center estimates for the two distributions. The effect of d' just missed significance at the .05 level. As would be expected, the greater the distance between the stimulus distributions in practice, the greater the mean distance between the Ss' estimates. For the three d' conditions from low to high the mean differences between estimates (across continua) were 2.34 in., 2.67 in., and 3.06 in., in terms of the dot position continuum. The ratio of the mean difference between estimates to the true distance between centers, however, decreased with increase in d', being $2.0,1.5$, and 1.3. The decrease in this ratio would be expected since the distance between the true centers increases with $d^{\prime}$, but the range of a continuum over which $\mathrm{S}$ could make his estimate remained constant. Therefore with increasing $d$ ' the range within which $S$ could overestimate the distance between centers became restricted. As in previous findings Ss overestimated the distance between the centers, and this was true for all d' conditions. The overestimation was significant for each $d^{\prime}$ based on the binomial test of the number of Ss who had estimated differences less than the true difference between centers.

Efficiencies. Table 1 shows the "efficiencies," defined in several ways, for the performance of the Ss broken down according to $d$ ' and continuum, and for all Ss combined versus learners. The different measures assist one's understanding of "how well" Ss performed, by approaching the question from several directions.

The basic data here are the probabilities of correct responses, taken as the proportion of the correct responses given by $\mathrm{Ss}$ for the last 200 trials. These values are shown in column (1). A response was scored to be correct if it was the same as the feedback given by the $E$ on a trial. This is to be distinguished from strategic correctness. The other measures of efficiency in the three columns after probability correct are all based on the probability correct figure in the first column. The second measure gives the ratio of probability correct to the probability of a correct response that would have been obtained had Ss used the optimal, i.e., the decision theory strategy. The third column gives 
Table 1. Mean Efficiency Values

\begin{tabular}{|c|c|c|c|c|c|c|}
\hline & Continuum & $d^{\prime}$ & $(1)^{a}$ & $(2)^{b}$ & $(3)^{c}$ & $(4)^{d}$ \\
\hline \multirow{7}{*}{ All Ss } & & $\mathrm{d} j$ & .64 & .88 & .63 & .37 \\
\hline & Dot & $d_{2}^{\prime}$ & .82 & .93 & .85 & .64 \\
\hline & & $d_{3}^{2}$ & .95 & .99 & .97 & .88 \\
\hline & & & & & & \\
\hline & & $d_{j}$ & .64 & .87 & .60 & .32 \\
\hline & Gray & $d_{2}^{\prime}$ & .77 & .83 & .74 & .44 \\
\hline & & $d_{3}^{\prime}$ & .88 & .92 & .83 & .45 \\
\hline \multirow{6}{*}{ Learners Only } & & $\mathrm{d}_{j}^{\prime}$ & .69 & .94 & .82 & .64 \\
\hline & Dot & $d_{2}^{\prime}$ & .84 & .96 & .92 & .77 \\
\hline & & $d_{3}^{\prime}$ & .95 & .99 & .97 & .88 \\
\hline & & & 68 & Q? & 76 & 55 \\
\hline & Gray & di & 83 & 94 & .83 & .69 \\
\hline & & $d_{3}^{2}$ & .93 & .97 & .93 & .72 \\
\hline \multirow{3}{*}{\multicolumn{2}{|c|}{ Decision Theory }} & $d \hat{j}$ & .73 & 1.00 & 1.00 & 1.00 \\
\hline & & $d_{2}^{\prime}$ & .87 & 1.00 & 1.00 & 1.00 \\
\hline & & $d_{3}^{2}$ & .96 & 1.00 & 1.00 & 1.00 \\
\hline \multirow{3}{*}{ Micromatching } & & $d_{i}$ & .64 & .88 & .63 & .37 \\
\hline & & $d_{2}^{\prime}$ & .81 & .93 & .83 & .59 \\
\hline & & $d_{3}^{\prime}$ & .94 & .98 & .95 & .76 \\
\hline
\end{tabular}

Note: $d_{1}^{\prime}=1.25, d_{2}^{\prime}=2.25, d_{3}^{\prime}=3.5$

a: Probability correct ( $P C_{a c t}$ )

b: $P C_{a c t} /$ probability correct if optimal strategy used $\left(P C_{o p t}\right.$

$c:\left(P C_{\text {act }}-.50\right) /\left(P C_{\text {opt }}-.50\right)$

$d:\left(d^{\prime}{ }_{\text {act }}^{\prime} d^{\prime}{ }_{t h}\right)^{2}$

the ratio of (a) increase of the probability correct over the probability correct that would have been obtained by random responding to (b) the increase over random response probability that could be obtained by decision theory. This figure has a lower bound of 0.0 and an upper bound of 1.0, whereas the second figure has an upper bound of 1.0 but a lower bound greater than .5 , which is a function of $d^{\prime}$. Thus relatively large values for measure (2) can be obtained under a random strategy, whereas for the measure (3) a random method of responding would give only a zero measure of efficiency. The last column is a measure of efficiency taken from detection theory (Swets, 1961), $\left(\mathrm{d}_{\mathrm{act}} / \mathrm{d}_{\mathrm{th}}\right)^{2}$. The actual $d^{\prime}$ is inferred from the percentage correct assuming Ss were using optimal strategy to get that percentage correct. The theoretical $d$ ' values were the nominal values $1.25,2.25$, and 3.5 . Thought of in terms of a communications system, this measure of efficiency gives the percentage of the energy available for discrimination which is utilized.

Note that if all Ss are considered for the dot position continuum, Ss perform slightly better than, but very close to micromatching. For all Ss for the gray continuum, Ss performed somewhat worse than micromatching. If only learners are considered for the dot continuum, Ss performed definitely better than micromatching, whereas, concerning only learners for the gray continuum, Ss performed somewhat better than micromatching. All in all, however, note that micromatching provides a pretty good description of the responses the Ss were making in this experiment, and that this statement remains true across the varying levels of $d^{\prime}$.

\section{Discussion}

The results of this study indicate that the micromatching hypothesis provides a fairly good description of Ss' behavior for different d' levels. The results of the present experiment correspond to micromatching more closely than the results of two previous experiments (Lee, 1963; Lee \& Janke, 1964), which gave results about midway between micromatching and decision theory. In this respect the present experiment resembles the finding of Lee \& Janke (1965). The difference may lie to a considerable extent in the instructions. In Lee (1963) Ss were told that the samples were randomly drawn from probability distributions, and were told the relative positions of the distributions. In Lee \& Janke (1964) Ss were told that "1's" were generally to the left of "2's" (fir the position continuum). In the present experiment, and in Lee \& Janke (1965), Ss were only told to guess what each stimulus card was, a "1" or a "2." One would think that it would become apparent that " 1 's" are generally to the left, but perhaps saying so is necessary for some Ss. A controlled experiment is necessary to tell for sure.

The increasing sharpness of the choice likelihood function with increasing $d^{\prime}$ substantiates with a different kind of evidence the conclusion of Lee \& Janke (1964) that the failure of Ss to use the sharp decision theory cutoff cannot be attributed simply to limitations on sensory discrimination; it must be considered to a large extent to be a learning phenomenon.

The null effect of having a $S$ give a confidence rating in addition to his response duplicates psycho, ysical results (Egan \& Clarke, 1956; Pollack \& Decker, 1958). Confidence ratings were employed in only one previous study in the present series (Lee, 1963). It is gratifying to have evidence that such ratings do not affect categorization responses.

The null effect of $\mathbf{E}$ variation was another gratifying result. This variable is seldom checked, but periodically it is suggested that $E$ variation may be implicated in failure to replicate experimental results.

Somewhat surprising was the lack of an effect of added payoff for improved performance. Such an improvement has been found a number of times for binary prediction tasks (Siegal \& Goldstein, 1959; Myers, Fort, Katz, \& Suydam, 1963), and the present task can be thought of as a set of simultaneous binary prediction tasks, each task being cued by a stimulus value of the continuum. In psychophysics, Swets \& Sewall (1963) found that there was some improvement in a detection task with added monetary motivation, and refer to other similar findings.

Do the results in the present experiment apply to psychophysical tasks? The present results by no means allow the assertion that micromatching, or some 
approximation to it, occurs in psychophysical experiments. For one thing, Ss enter such experiments with an understanding of attributes such as loudness, whereas in the present experiment Ss begin without any idea of how to respond to what stimulus. For another, in psychophysical experiments Ss may have to be concerned with problems of sensory attention, to the pitch of the tone, for example.

Still, consider that there was a time when the Ss did not know what "there is a sound present" meant, and for soft sounds the reinforcement was only probabilistic, particularly so in relation to their internal continua. Then they could have learned to respond probabilistically to a given internal signal, just as they came to a steady mode of probabilistic responding in the present task. Of course the distributions for "noise" and "signal" were more separated in real life than in the present learning task, and thus there are no nonlearners for "loudness." When first entering the laboratory, and if given no feedback, Ss may match to reinforcements given in real life. If feedback is given in the laboratory, this may result in "re-learning." Treatments of learning processes in psychophysics from the viewpoint of statistical learning theory are provided by Atkinson (1963), Luce (1963), Schoeffler (1965), and Lee (1966). The latter two papers consider a continuous internal representation, as does detection theory.

An experimental technique that might be useful for shedding light on the relationship between externally distributed stimuli tasks, and psychophysical tasks, would be to present, e.g., a standard detection task to one group, and an abstract version of the task to another group. This latter group would not be told that presence or absence of a signal was the critical determinant of the correct response. They would be given feedback after each trial, though, stating whether " 1 " or "2" was the correct response for that trial. If there were any difference in performance of the two groups, one could conclude that the abstract method of presentation does affect final performance, and that the abstract nature of the task in the present experiment critically distinguishes the task from the analogous psychophysical tasks.

The improvement of performance for the low d' condition with the removal of feedback, may at first seem a-intuitive, since "information" should allow superior performance, not inferior. Howdver, Bruner, Goodnow, \& Austin (1956, pp. 208-216) discuss the effect of reduced opportunity for validation in probabilistic categorization tasks, and theorize that reduced opportunities for validation will result in a more nearly optimal performance. They present evidence that this indeed happens. With reduced opportunities for valida- tion (i.e., reduced feedback) the hopeless attempt to eliminate error is forsaken, in favor of $\mathrm{rr}$;ponding according to the most probable answer. If, for example, Ss have been attempting to base their responses fallaciously on the feedback to a given cue on a particular trial, then when such feedback is eliminated, such a fallacious method of responding can no longer be utilized.

Although improvement of response strategy with elimination of feedback is not an entirely idiosyncratic result, it does not correspond to usual results in psychophysics, where feedback has been found to have either slight effect, or to improve performance (Gundy, 1961; Lukaszewski \& Elliot, 1961).

\section{References}

Atkinson, R. C. A variable sensitivity theory of signal detection. Psychol. Rev., 1963, 70, 91-106.

Bruner, J. S., Goodnow, J. J., \& Austin, G. A. A study of thinking. New York: Wiley, 1956.

Egan, J. P., \& Clarke, F. R. Source and receiver behavior in the use of a criterion. J. Acoust. Soc. Amer., 1956, 28, 1267-1269.

Gundy, R. F. Auditory detection of an unspecified signal. $J$. Acoust. Soc. Amer., 1961, 33, 1008-1012.

Lee, W. Choosing among confusably distributed stimuli with specified likelihood ratios. Percept. mot. Skills, 1963, 16, 445-467.

Lee, $W$. Conditioning parameter model for reinforcement generalization in probabilistic discrimination learning. J. math. Psychol., 1966, 3, in press.

Lee, W, \& Janke, M. Categorizing externally distributed stimulus samples for three continua. $J$, exp. Psychol., 1964, 68, 376-382.

Lee, W., \& Janke, M. Categorizing externally distributed stimulus samples for unequal molar probabilities. Psychol. Rep., 1965. $17,79-90$.

Luce, R. D. A threshold theory for simple detection experiments. Psychol. Rev., 1963, 70, 61-79.

Lukaszewski, J. S., \& Elliot, D. N. Auditory threshold as a function of forced-choice techniques, feedback, and motivation. $J$. Acoust. Soc. Amer., 1962, 34, 223-228.

Myers, J. L., Fort, J. G., Katz, L., \& Suydam, M. M. Differential monetary gains and losses and event probability in a two-choice situation. J. exp. Psychol., 1963, 66, 521-522.

Pollack, I., \& Decker, L. R. Confidence ratings, message reception, and the receiver operating characteristic. J. Acoust. Soc. Amer., 1958, 30, 286-292.

Schoeffler, M. S. Theory for psychophysical learning. J. Acoust. Soc. Amer., 1965, 37, 1124-1133.

Siegel, S., \& Goldstein, D. A. Decision-making behavior in a two-choice uncertain outcome situation. J. exp. Psychol., 1959, $57,37-42$.

Swets, J. A. Is there a sensory threshold? Science, 1961, 134, 168-177.

Swets, J. A, \& Sewall, S. T. Invariance of signal detectability over stages of practice and levels of motivation. J. exp. Psychol., 1963, 66, 120-126.

\section{Note}

1. This research was supported by NIH Grant 11128, and was facilitated by grants by NSF and NIH to the Institute of Human Learning. Mr. Michael E. Gordon assisted us in running Ss, and wrote a computer program used in data analysis.

(Received in the Editorial Office February 8, 1966.) 